\title{
KNOW THE STAR, KNOW THE PLANET. II. A STELLAR COMPANION TO THE HOST STAR OF THE ECCENTRIC EXOPLANET HD 8673B
}

\author{
Lewis C. Roberts, Jr. ${ }^{1}$, Brian D. Mason ${ }^{2}$, Christopher R. Neyman ${ }^{3}$, Yanqin Wu ${ }^{4}$, Reed L. Riddle ${ }^{5}$, \\ J. Christopher Shelton ${ }^{1}$, John Angione ${ }^{1,12}$, Christoph Baranec ${ }^{6}$, Antonin Bouchez ${ }^{7,8}$, Khanh Bui ${ }^{5}$, Rick Burruss ${ }^{1}$, \\ Mahesh Burse ${ }^{9}$, Pravin Chordia ${ }^{9}$, Ernest Croner ${ }^{5}$, Hillol Das ${ }^{9}$, Richard G. Dekany ${ }^{5}$, Stephen Guiwits ${ }^{10}$, David Hale ${ }^{5}$, \\ John Henning ${ }^{5}$, Shrinivas Kulkarni ${ }^{5}$, Nicholas Law ${ }^{11}$, Dan McKenna ${ }^{5}$, Jennifer Milburn ${ }^{5}$, Dean Palmer ${ }^{1}$, \\ Sujit Punnadi ${ }^{9}$, A. N. Ramaprakash ${ }^{9}$, Jennifer E. Roberts ${ }^{1}$, Shriharsh P. Tendulkar ${ }^{5}$, Thang Trinh $^{1}$, Mitchell Troy ${ }^{1}$, \\ Tuan Truong ${ }^{1}$, AND JefF ZolKower ${ }^{5}$ \\ ${ }^{1}$ Jet Propulsion Laboratory, California Institute of Technology, 4800 Oak Grove Drive, Pasadena CA 91109, USA; lewis.c.roberts@jpl.nasa.gov \\ ${ }^{2}$ U.S. Naval Observatory, 3450 Massachusetts Avenue, NW, Washington, DC 20392-5420, USA \\ ${ }^{3}$ W. M. Keck Observatory, California Association for Research in Astronomy, 65-1120 Mamalahoa Hwy, Kamuela, HI 96743, USA \\ ${ }^{4}$ Department of Astronomy and Astrophysics, University of Toronto, ON M5S 3H4, Canada \\ ${ }^{5}$ Division of Physics, Mathematics, and Astronomy, California Institute of Technology, Pasadena, CA 91125, USA \\ ${ }^{6}$ Institute for Astronomy, University of Hawai'‘ at Mānoa, Hilo, HI 96720-2700, USA \\ ${ }^{7}$ GMTO Corp., 251 S. Lake Ave., Pasadena, CA 91101, USA \\ ${ }^{8}$ Observatories of the Carnegie Institution for Science, 813 Santa Barbara St., Pasadena, CA 91101, USA \\ 9 Inter-University Centre for Astronomy \& Astrophysics, Ganeshkhind, Pune, 411007, India
${ }^{10}$ Seismological Laboratory, California Institute of Technology, 1200 E. California Blvd., Pasadena, CA 91101, USA \\ ${ }^{11}$ Department of Physics and Astronomy, University of North Carolina at Chapel Hill, Chapel Hill, NC 27599-3255, USA \\ Received 2014 June 20; accepted 2015 February 25; published 2015 March 30
}

\begin{abstract}
HD 8673 hosts a massive exoplanet in a highly eccentric orbit $(e=0.723)$. Based on two epochs of speckle interferometry a previous publication identified a candidate stellar companion. We observed HD 8673 multiple times with the $10 \mathrm{~m}$ Keck II telescope, the $5 \mathrm{~m}$ Hale telescope, the $3.63 \mathrm{~m}$ Advanced Electro-Optical System telescope, and the $1.5 \mathrm{~m}$ Palomar telescope in a variety of filters with the aim of confirming and characterizing the stellar companion. We did not detect the candidate companion, which we now conclude was a false detection, but we did detect a fainter companion. We collected astrometry and photometry of the companion on six epochs in a variety of filters. The measured differential photometry enabled us to determine that the companion is an early $\mathrm{M}$ dwarf with a mass estimate of $0.33-0.45 M_{\odot}$. The companion has a projected separation of $10 \mathrm{AU}$, which is one of the smallest projected separations of an exoplanet host binary system. Based on the limited astrometry collected, we are able to constrain the orbit of the stellar companion to a semimajor axis of $35-60 \mathrm{AU}$, an eccentricity $\leqslant 0.5$, and an inclination of $75^{\circ}-85^{\circ}$. The stellar companion has likely strongly influenced the orbit of the exoplanet and quite possibly explains its high eccentricity.
\end{abstract}

Key words: binaries: visual - instrumentation: adaptive optics - stars: individual (HD 8673) - stars: late-type

\section{INTRODUCTION}

HD $8673($ HR $410=$ HIP $6702=$ WDS 01262+3435) is identified as a member of the larger Hyades Moving Group although it does not fit the Hyades Li-temperature pattern or the Hyades Fe abundances (Boesgaard \& Budge 1988). There is a spread in the age estimates of the star. Saffe et al. (2005) derived an age of $2.8_{-0.7}^{+0.5} \mathrm{Gyr}$ from isochrones and an age of $8.7 \mathrm{Gyr}$ from $\mathrm{Fe} / \mathrm{H}$ abundances. Holmberg et al. (2009) produced a similar age of $2.5 \mathrm{Gyr}$ using the photometric technique. Using isochrones, Valenti \& Fischer (2005), computed an age of 4.29 Gyr. This variation in age estimates is normal due to a number of factors (Saffe et al. 2005) and HD 8673 is probably somewhere in age between the Hyades and the Sun. It can be broadly described as a nearby solar type star. It was spectroscopically classified as an F7V (Boesgaard \& Friel 1990) and has a distance of $36.1 \pm 0.5 \mathrm{pc}$ (van Leeuwen 2007). Tsantaki et al. (2014) computed that it had a $T_{\text {eff }}$ of $6472 \pm 64$, a mass of $1.56 \pm 0.10 M_{\odot}$, and a radius of $1.23 \pm 0.07 R_{\odot}$.

12 Deceased.
A substellar companion orbiting HD 8673 was detected via radial velocity (RV) measurements by Hartmann et al. (2010). HD $8673 \mathrm{~b}$ has a minimum mass of $14.2 \pm 1.6 M_{\text {Jup }}$, suggested that it is probably a low-mass brown dwarf rather than a planet. The companion is in a high eccentricity $(e=0.723 \pm 0.016)$ orbit with a period of $1634 \pm 17$ days. The orbit has a semimajor axis of $3.02 \pm 0.15 \mathrm{AU}$. The detection was part of an effort to detect sub-stellar companions to F-type stars in order to increase the statistics of exoplanets around stars more massive than the Sun.

In an earlier paper, we described a candidate stellar companion to HD 8673 (Mason et al. 2011). In order to confirm the candidate companion and determine the impact of the stellar companion on the orbital dynamics of the planetary system, we conducted a series of adaptive optics (AO) observations using four different telescopes. Those observations now lead us to conclude that our previous detection was spurious. However, we detected another companion that appears to be a bound low-mass stellar companion in a century-long orbit. Details of the observations are in Section 2, and the analysis of the companion is detailed in Section 3. Finally we discuss the system in Section 4. 
Table 1

Astrometry

\begin{tabular}{lccl}
\hline \hline UT & $\theta\left(^{\circ}\right)$ & $\rho\left(^{\prime \prime}\right)$ & Telescope \\
\hline 2004.7871 & $302.3 \pm 1.0$ & $0.31 \pm 0.02$ & AEOS \\
2011.5366 & $332.3 \pm 1.0$ & $0.310 \pm 0.005$ & Keck II \\
2011.6402 & $329.5 \pm 1.0$ & $0.308 \pm 0.1$ & Hale \\
2011.8671 & $333.4 \pm 0.57$ & $0.308 \pm 0.003$ & Keck II \\
2012.4837 & $335.2 \pm 0.63$ & $0.308 \pm 0.003$ & Keck II \\
2013.7426 & $339.3 \pm 1.68$ & $0.32 \pm 0.02$ & Hale \\
\hline
\end{tabular}

\section{OBSERVATIONS}

We observed HD 8673 with four telescopes, each equipped with AO. Two of the AO systems operate in the visible, while two operate in the near-IR.

\subsection{Keck II Observations}

We used the Keck II telescope, its AO system (Wizinowich et al. 2000), and the NIRC2 instrument to observe the system at three epochs in 2011 and 2012. Each time, we collected data in multiple filters with multiple coadds. Since the existence of the companion was still questionable, the multiple filters allowed us to determine if the object was an astronomical object or a quasi-static speckle whose position is wavelength dependent. The $2012 \mathrm{Br} \gamma$ image of HD 8673 and its companion is shown in Figure 1.

After reducing each image, we measured the astrometry and photometry using the fitstars algorithm on each image (ten Brummelaar et al. 1996, 2000). Photometric error bars were set equal to the standard deviation of the measurements from all the images. We computed the weighted mean of the astrometry from the images taken in different filters on the same night, with the weight being the number of coadds for each image. The error bar was set to the standard deviation of the results. That covers much of the random errors, but does not cover the systematic errors. We expect those to be smaller than those of the Advanced Electro-Optical System (AEOS; Section 2.3) or Hale data (Section 2.2) because of the smaller point-spread function (PSF) arising from the larger aperture size, but we do not have a way of quantifying those errors. The astrometry is given in Table 1; it lists the Besselian date of the observations, the position angle and separation of the companion relative to the primary and the telescope used to collect the measurements. The photometric measurements are listed in Table 2. The table lists the measured magnitude difference in each filter, the central wavelength of those filters, and the telescope used to take the observations.

\subsection{Hale Observations}

We observed HD 8673 on 2011 August 22 UT and 2013 September 28 UT with the Palomar Observatory Hale $5 \mathrm{~m}$ telescope using the PALM 3000 AO system (Dekany et al. 2013) and the PHARO near-IR camera (Hayward et al. 2001). HD 8673 was centered in the detector's 25" field of view. In 2011, we collected 10 frames of the star in $\mathrm{Br} \gamma$ filter and after reducing the data, the frames were coadded. In 2013, we collected 50 frames in the $K_{s}$ filter. After the individual frames were calibrated, we created five images by coadding 10 frames into each image, allowing us to analyze the precision of the measurements. The fitstars algorithm was used to measure the astrometry and photometry of the objects. Photometric error bars were assigned using the technique described in Roberts et al. (2005). The resulting astrometry and photometry are listed in Tables 1 and 2. Figure 2 shows an image from the 2013 data collection.

\subsection{AEOS Observations}

Following the Keck II and Hale resolutions, archival observations obtained with the AEOS $3.67 \mathrm{~m}$ telescope (Vigil et al. 1996) were re-examined for a comparable companion detection. On 2004 October 14 UT the star was observed in an effort to detect the bright companion detected by Mason et al. (2011). The observations used the telescope's AO system and the Visible Imager camera (Roberts \& Neyman 2002). We collected 1000 frames of the star with the Bessel I filter. After debiasing, dark subtraction, and flat fielding, the resulting frames were coadded with each frame weighted by its peak pixel to emphasize the best quality data. The result is shown in Figure 3. The companion is harder to see than in the Keck II or Hale images, due to a lower image quality because of the shorter wavelength and because the system has a larger dynamic range in the $I$ filter.

Again, we measured the astrometry and photometry with the iterative blind deconvolution algorithm, fitstars. Photometric error bars were assigned using the technique described in Roberts et al. (2005). Tables 1 and 2 list the resulting astrometry and photometry measurements.

\subsection{Palomar 1.5 M Observations}

In 2012 and 2013, we observed HD 8673 four times with the Robo-AO system (Baranec et al. 2013, 2014) on the Palomar $1.5 \mathrm{~m}$ telescope. On 2012 July 17 UT and 2012 July 18 UT, we used the SDSS r', $i$, $z$ ' filters, on 2012 October 6 UT we used the SDSS $i$ ' and $g$ ' filters, and on 2013 January 20 UT we used the SDSS r', $i$ ', $z$ ' filters. In 2012, the observations were collected as part of the automated Robo-AO target queue, where the telescope is pointed at the object robotically with a $44^{\prime \prime}$ wide square field of view. For the 2013 January observations, the star was acquired manually which allowed the star to be positioned at the exact center of camera's field of view, and a 5'.5 sub window of the image to be read out at $50.5 \mathrm{~Hz}$, which is 5 times faster than the standard rate for the full image. This improves the image quality by reducing the uncompensated intra-exposure stellar image motion. Capturing data in this manner is done infrequently, as it requires human intervention and drastically slows the acquisition rate.

Each data set was reduced using the standard Robo-AO lucky imaging pipeline to perform the image alignment (Terziev et al. 2013; Law et al. 2014). The pipeline produces four images, with each image selecting either the best 1, 10, 50 or $100 \%$ of the images. Each image was examined visually for companions and no companions were detected. We computed the dynamic range of the image via the method of Turner et al. (2008) and determined that the images have insufficient dynamic range to detect the companion. We also examined the wider field of view image taken in 2012 and were able to rule out any additional companions to a limit of 10 magnitudes fainter than the primary. 
Table 2

Photometry

\begin{tabular}{|c|c|c|c|c|c|c|c|}
\hline \multicolumn{8}{|c|}{ Magnitude Difference } \\
\hline UT & $\begin{array}{c}I \\
(0.81 \mu \mathrm{m})\end{array}$ & $\begin{array}{c}J c \\
(1.21 \mu \mathrm{m})\end{array}$ & $\begin{array}{c}H c \\
(1.58 \mu \mathrm{m})\end{array}$ & $\begin{array}{c}\mathrm{Fe}_{\mathrm{II}} \\
(1.65 \mu \mathrm{m})\end{array}$ & $\begin{array}{c}B r \gamma \\
(2.15 \mu \mathrm{m})\end{array}$ & $\begin{array}{c}K s \\
(2.17 \mu \mathrm{m})\end{array}$ & Telescope \\
\hline 2004.7871 & $6.5 \pm 1.0$ & $\ldots$ & $\cdots$ & $\cdots$ & $\cdots$ & $\cdots$ & AEOS \\
\hline 2011.6402 & $\ldots$ & $\ldots$ & $\ldots$ & $\ldots$ & $4.50 \pm 0.6$ & $\ldots$ & Hale \\
\hline 2011.8671 & $\cdots$ & $4.56 \pm 0.02$ & $4.20 \pm 0.03$ & $4.07 \pm 0.06$ & $3.98 \pm 0.08$ & $\cdots$ & Keck II \\
\hline 2012.4837 & $\cdots$ & $\cdots$ & $4.12 \pm 0.06$ & $4.03 \pm 0.06$ & $3.97 \pm 0.09$ & $\cdots$ & Keck II \\
\hline
\end{tabular}

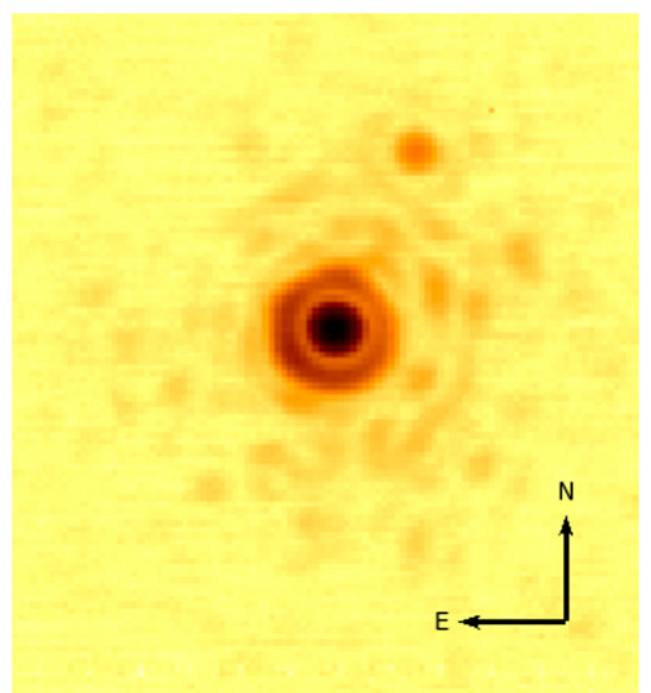

Figure 1. Br $\gamma$ image of the HD 8673 binary system taken with NIRC2 at the Keck II telescope on 2012 June 12. North is up in the image and east is to the left. This is a sub-image of the full image and is approximately 1.2 across.

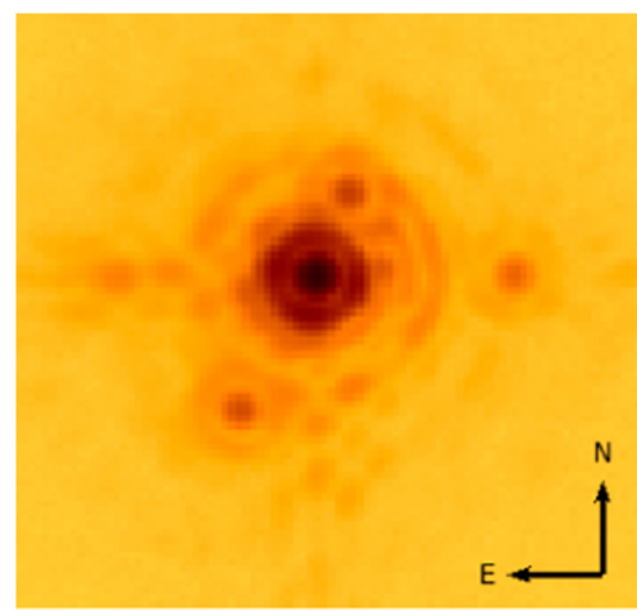

Figure 2. $K s$ image of the HD 8673 binary system taken with PHARO an "d the PALM 3000 AO system at the Hale telescope on 2013 September 28 UT. The companion is the object to the upper right. There is a ghost image caused by the neutral density filter to the lower left. There is a large speckle to the right of the primary and a mirror reflection of that speckle to the left of the primary. The image has the same orientation as Figure 1. This is a sub-image of the full $25^{\prime \prime}$ field of view and is approximately 2.5 across.

\section{ANALYSIS}

\subsection{Proper Motion}

Between 2011 and 2012 the measurements from Keck II show the companion moved at a rate of $16.47 \mathrm{mas} \mathrm{yr}^{-1}$ compared to the measured proper motion of HD 8673 of $251.8 \mathrm{mas} \mathrm{yr}^{-1}$ (van Leeuwen 2007). The low relative motion of the companion indicates that it shares common proper motion with the primary star and is not a background object. The Keck II data were used to measure the relative proper motion since it is a collection of data taken with the same instrument. The AEOS and Hale data are also consistent with it being a bound object.

There is very little difference in the short term proper motion $\left(\alpha=237.19 \pm 0.33\right.$ mas yr$^{-1}, \delta=-84.64 \pm 0.25 \mathrm{mas} \mathrm{yr}^{-1}$; van Leeuwen 2007) and the long term proper motion $\left(\alpha=237.9 \pm 1.0\right.$ mas $\mathrm{yr}^{-1}, \quad \delta=-87.2 \pm 1.1 \mathrm{mas} \mathrm{yr}^{-1}$, $\mathrm{Høg}$ et al. 2000). Significant differences in these values can indicate the presence of companions (Makarov \& Kaplan 2005), but their absence is not surprising, especially in the case of a pair with a large differential magnitude and hence large mass ratio.

\subsection{Comparision With Prior Observations}

The two observations of the companion reported by Mason et al. (2011) were both optained with visible speckle interferometry using an intensified CCD camera. The observations were in the Strömgren $y$ filter $(550 \pm 24 \mathrm{~nm})$ and the $\Delta m$ from the 2007 measure is estimated as $2.3 \pm 0.5$. The quality of the 2001 measure was insufficient for an estimation of the differential magnitude. Using the same speckle interferometry camera as Mason et al. (2011), Hartkopf \& Mason (2009) did not detect the companion in observations using the Mt. Wilson $2.5 \mathrm{~m}$ telescope on 2007.8179 , only a short time after the 2007.6049 detection in Mason et al. (2011). The observations had a limiting resolution of $0 . \prime 054$. This would have been sufficient to detect the 0.'109 separation detected by Mason et al. (2011) measured just a few months earlier. In addition, no companions were detected by Ginski et al. (2012) using the $2.2 \mathrm{~m}$ Calar Alto telescope and the AstraLux lucky imaging camera in the SDSS $i$ ' filter on 2011 January 14.

Our observations using $\mathrm{AO}$ have a higher dynamic range than speckle interferometry and would have detected any companions detected with that technique, even if those companions had considerable motion. The astrometry in Table 1 is inconsistent with the companion reported in Mason et al. (2011). Our non-detection of the candidate companion from Mason et al. (2011) and the non-detection in Hartkopf \& Mason (2009) and Ginski et al. (2012) lead us to conclude that the Mason et al. (2011) detection was spurious. This is not the only known case of false doubles in speckle interferometry; see McAlister et al. (1993) and Tokovinin (2012) for examples and further discussion. 


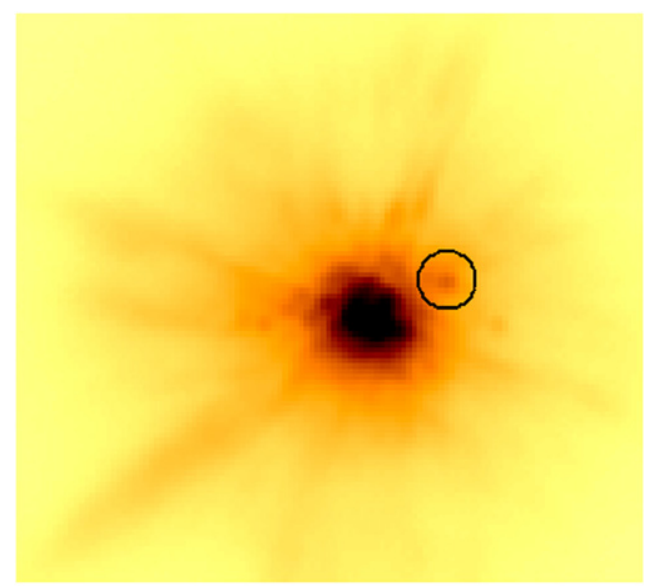

Figure 3. I-band image of HD 8673 collected with the AEOS telescope and AO system on 2004 October 14 UT. The companion is circled. The halo of the PSF has many more speckles than the near-IR images, but that is to be expected due to the shorter wavelength of the AEOS images. The image has the same orientation as Figures 1 and 2. This is a sub-image of the full $10^{\prime \prime}$ image and is roughly 1.8 wide.

The companion reported in this paper is a redder object than the purported object in Mason et al. (2011). Visible speckle interferometry using intensified CCD cameras can only detect companions with differential magnitudes less than 3.5 (Mason 1996). This is too low to detect the companion reported in this paper and this explains why it was not seen by Hartkopf \& Mason (2009), or Mason et al. (2011). The observations of Ginski et al. (2012) also did not have sufficient dynamic range to detect the companion.

\subsection{Photometric Analysis}

Based on the distance (van Leeuwen 2007) to HD 8673 AB and the I-band apparent magnitude (Monet et al. 2003), we computed the absolute magnitude of HD $8673 \mathrm{~B}$ in $I$ band of $9.5 \pm 0.94$. Using the 2MASS apparent magnitudes in $J, H$, and $K$ (Skrutskie et al. 2006), we also computed the absolute magnitudes of companion in the $J, H$ and $K$ filters. Our data were taken in $J c, H c$, and $K s$ and we do not have a transformation between these filters into $J, H$, and $K$. Instead we use the values from narrow band filters and realize that there is an unestimated error in the magnitudes. We compared the resulting absolute magnitudes to the values listed on the Dartmouth Stellar Evolution Database ${ }^{13}$ (Dotter et al. 2008) for a 3 Gyr old star (Saffe et al. 2005) with an $\mathrm{Fe} / \mathrm{H}$ of -0.01 (Nordström et al. 2004). The $J, H, K s$ absolute magnitudes all fall in the same bin. This bin has a mass of $0.33-0.45 M_{\odot}, T_{\text {eff }}$ of $3520-3690 \mathrm{~K}$, and $\log G$ of $4.94-4.85$. The $I$ absolute magnitude has a much larger error bar and corresponds to a mass range of $0.17-0.44 M_{\odot}$. There is some overlap between the infrared and the $I$ data. This variance in the $I$-band result is not surprising. The $I$ data is the most error prone of all the measurements. As seen in Figure 3 the secondary PSF is in the halo of the primary; it also has the largest dynamic range.

\section{DISCUSSION}

While the astrometric data shown in Table 1 does not cover enough of the orbit to yield a uniqe orbital solution, it does

\footnotetext{
13 http://stellar.dartmouth.edu/models
}

allow us to place some constraints on the orbital semimajor axis, eccentricity and inclination. The results are shown in Figure 4. We detail our procedure here, followed by a short discussion on the implications.

Based on the spectral class of the primary and the secondary, F7V and M2V, we obtain a mass sum of $\sim 1.8 M_{\odot}$ (masses of 1.4 and $0.4 M_{\odot}$, respectively). Adopting this total mass, we then search through the parameter spaces of all six orbital elements to look for acceptable solutions. Due to the shortness of the arc, there is a wide range of orbital elements that lead to $\chi_{\text {reduced }}^{2} \leqslant 1$, except for orbital inclinations, which are strongly constrained to be between $75^{\circ}$ and $85^{\circ}$ from face-on. The binary orbit is nearly edge-on, and the companion is now lying nearly along our line of sight to the star. This fact can be simply inferred, without detailed orbital fitting, from the apparent angular motion of the orbit $\left(\sim 4^{\circ} \mathrm{yr}^{-1}\right)$, the apparent separation $(\sim 0 . \prime 3$, or $11 \mathrm{AU}$ at a distance of $36 \mathrm{pc})$, and the system's total mass.

We can pare down possible orbital solutions using two further constraints. The first is the lack of evidence for $\mathrm{RV}$ residuals from the binary companion in the Hartmann et al. (2010) measurements (over $6.3 \mathrm{yr}$ ). The RV residuals, after subtracting the signals from the highly eccentric planet, is shown to be nearly flat. We adopt a conservative limit of $\Delta \mathrm{RV} \leqslant 200 \mathrm{~m} \mathrm{~s}^{-1}$ over $6.3 \mathrm{yr}$, or $\Delta \mathrm{RV} \leqslant 285 \mathrm{~m} \mathrm{~s}^{-1}$ over $9 \mathrm{yr}$ (the astrometry time-span). The second, more important, constraint arises from the presence of the eccentric planetthe binary orbit ought not to destabilize the planet $\left(a_{p}=3.02 \mathrm{AU}, e_{p}=0.723\right)$. This constraint, as we show below, rules out a large swath of binary orbits.

Holman \& Wiegert (1999) numerically investigated the stability of a planet orbiting around a star that has a stellar companion. In their simulations, the planet was initially on a circular, coplanar orbit. For a binary mass ratio of 0.28 (the case here), and a typical binary eccentricity of 0.5 , the binary has to have a semimajor axis $a_{B} \geqslant 7.1 a_{p}$ for stability. This corresponds to $a_{B} \geqslant 22 \mathrm{AU}$. However, our planet has a high eccentricity, and is possibly not coplanar with the binary orbit. To account for this, we perform our own numerical simulations to define the stability boundary and the results, for coplanar orbits, are also shown in Figure 4. These allow us to exclude all orbits with $a_{B} \leqslant 35 \mathrm{AU}$. The acceptable orbits that meet all these criteria are shown in Figure 5.

While the astrometry data do not exclude orbits that are very wide (e.g., $a_{B} \geqslant 60 \mathrm{AU}$ ), these are statistically less probable because the chance of catching the companion at the small separation of $11 \mathrm{AU}$ is smaller. Roughly, we can peg the most likely binary orbits as $a_{B} \in[35,60] \mathrm{AU}$ and $e_{B} \leqslant 0.5$.

This brings us to the question of whether the binary companion has contributed to the planet's high eccentricity. Unless the binary orbit is wide $\left(a_{B} \sim 60 \mathrm{AU}\right)$ and circular, simulations show the companion strongly influences the planet's orbit. This is even more so if the two orbits are substantially inclined to each other. It is conceivable that HD $8673 \mathrm{~b}$ was born with a low eccentricity, but is pumped to its current value by the binary companion. It is also conceivable that the high mass of HD $8673 \mathrm{~b}\left(M \sin i=14.2 M_{J}\right)$ is related to this perturbatio- the large eccentricity swing of the planet may have allowed it to sweep through, and accrete from, a larger area of the protoplanetary disk.

In conclusion, the discovery of the companion of HD 8673 may have provided us with an opportunity to study the impact 


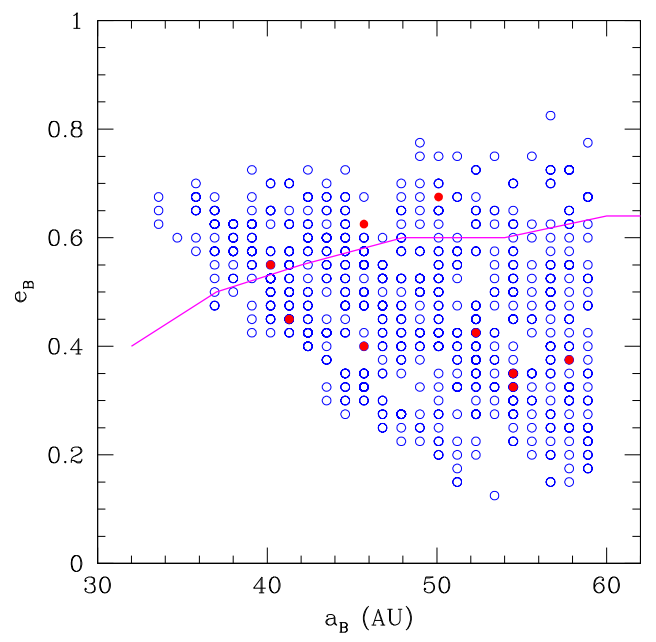

Figure 4. All binary orbits that satisfy the astrometry constraints (Table 1) to within $\chi_{\text {reduced }}^{2} \leqslant 4$ (blue open circles) and $\chi_{\text {reduced }}^{2} \leqslant 1$ (red filled circles), obtained by scanning through all orbital elements. All these orbits are close to edge-on (inclination between $75^{\circ}$ and $85^{\circ}$ ). Only orbits that avoid RV detection are shown (see text). Orbits that lie above the magenta lines (for anti-aligned apses between the planet and the binary orbits) will destabilize the highly eccentric planet, HD 8673b. A non-coplanar situation will also move the magenta lines downward. Admissable binary solutions have semimajor axis $a_{B} \geqslant 35 \mathrm{AU}$. Orbits with very large $a_{B}$ are disfavored.

of binarity on planet formation and migration. There is still much work to be done on the system. Additional astrometric observations over the next five to ten years are needed to pin down the orbit of the stellar companion. It would also be interesting to collect additional RV data to see if there is an RV acceleration caused by the stellar companion. This approach has been fruitful in discovering low mass companions (Crepp et al. 2012; Knutson et al. 2014). An RV acceleration would further constrain the orbit. An improved orbit will allow us to quantitatively retrace the dynamical history of the system.

The research in this paper was carried out at the Jet Propulsion Laboratory, California Institute of Technology, under a contract with the National Aeronautics and Space Administration. This paper is based on observations from a number of observatories, including the Maui Space Surveillance System, operated by the US Air Force Research Laboratory's Directed Energy Directorate. Additional observations were made at the Hale Telescope, Palomar Observatory as part of a continuing collaboration between the California Institute of Technology, NASA/JPL, NOAO, Oxford University, Stony Brook University, and the National Astronomical Observatories of China. Some of the data presented herein were obtained at the W.M. Keck Observatory, which is operated as a scientific partnership among the California Institute of Technology, the University of California and the National Aeronautics and Space Administration. The W.M. Keck Observatory was made possible by the generous financial support of the W.M. Keck Foundation. The Robo-AO system is supported by collaborating partner institutions, the California Institute of Technology and the Inter-University Centre for Astronomy and Astrophysics, by the National Science Foundation under grant Nos. AST0906060, AST-0960343, and AST-1207891, by a grant from the Mt. Cuba Astronomical Foundation and by a gift from
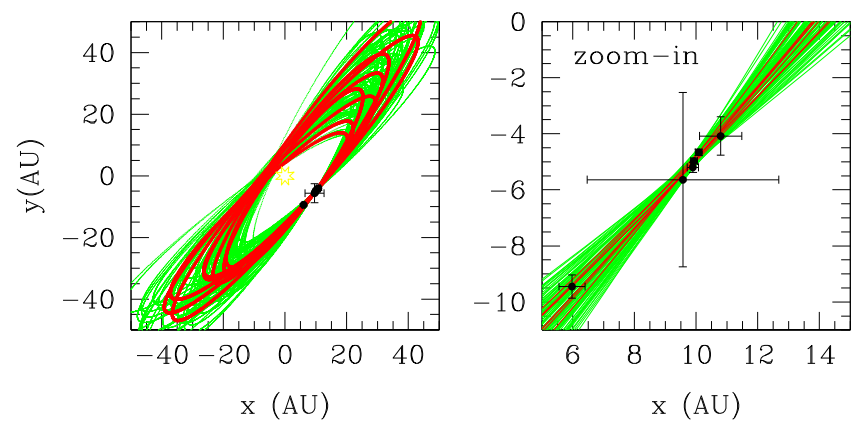

Figure 5. Orbital fit for the acceptable solutions in Figure 4 where red ones satisfy $\chi^{2} \leqslant 1$, and green ones $1 \leqslant \chi^{2} \leqslant 2$. The binary orbit has to be nearly edge-on (with inclination angles between $75^{\circ}$ and $85^{\circ}$, in order to satisfy the astrometry observations (points with error-bars).

Samuel Oschin. C.B. acknowledges support from the Alfred P. Sloan Foundation. This research made use of the Washington Double Star Catalog maintained at the U.S. Naval Observatory, the SIMBAD database, operated by the CDS in Strasbourg, France, NASA's Astrophysics Data System and data products from the 2MASS, which is a joint project of the University of Massachusetts and the Infrared Processing and Analysis Center/California Institute of Technology, funded by the National Aeronautics and Space Administration and the National Science Foundation.

Facilities: AEOS (Visible Imager), Hale (PHARO), Keck:II (NIRC2), PO:1.5 m (Robo-AO).

\section{REFERENCES}

Baranec, C., Riddle, R., Law, N., et al. 2013, J. Vis. Exp., 72, e50021

Baranec, C., Riddle, R., Law, N., et al. 2014, ApJL, 790, L8

Boesgaard, A. M., \& Friel, E. D. 1990, ApJ, 351, 467

Boesgaard, A. M., \& Budge, K. G. 1988, ApJ, 332, 410

Crepp, J. R., Johnson, J. A., Howard, A. W., et al. 2012, ApJ, 761, 39

Dekany, R. G., Burruss, R., Shelton, J. C., et al. 2013, ApJ, 776, 130

Dotter, A., Chaboyer, B., Jevremović, D., et al. 2008, ApJS, 178, 89

Ginski, C., Mugrauer, M., Seeliger, M., \& Eisenbeiss, T. 2012, MNRAS, 421, 2498

Hartkopf, W. I., \& Mason, B. D. 2009, AJ, 138, 813

Hartmann, M., Guenther, E. W., \& Hatzes, A. P. 2010, ApJ, 717, 348

Hayward, T. L., Brandl, B., Pirger, B., et al. 2001, PASP, 113, 105

Høg, E., Fabricius, C., Makarov, V. V., et al. 2000, A\&A, 355, 27

Holman, M. J., \& Wiegert, P. A. 1999, AJ, 117, 621

Holmberg, J., Nordström, B., \& Andersen, J. 2009, A\&A, 501, 941

Jones, H. R. A., Butler, R. P., Tinney, C. G., et al. 2006, MNRAS, 369, 249

Kley, W., \& Nelson, R. P. 2008, A\&A, 486, 617

Knutson, H. A., Fulton, B. J., Montet, B. T., et al. 2014, ApJ, 785, 126

Law, N. M., Morton, T., Baranec, C., et al. 2014, AJ, 491, 35

Lithwick, Y., \& Wu, Y. 2014, PNAS, 111, 12610

Macintosh, B. A., Anthony, A., Atwood, J., et al. 2012, Proc. SPIE, 8446, $84461 \mathrm{U}$

Makarov, V. V., \& Kaplan, G. H. 2005, AJ, 129, 2420

Mason, B. D. 1996, PhD Disseration, Georgia State Univ.

Mason, B. D., Hartkopf, W. I., Raghavan, D., et al. 2011, AJ, 142, 176

McAlister, H. A., Mason, B. D., Hartkopf, W. I., \& Shara, M. M. 1993, AJ, 106, 1639

Monet, D. G., Levine, S. E., Canzian, B., et al. 2003, AJ, 125, 984

Nordström, B., Mayor, M., Andersen, J., et al. 2004, A\&A, 418, 989

Roberts, L. C., Jr., \& Neyman, C. R. 2002, PASP, 114, 1260

Roberts, L. C., Jr., Turner, N. H., Bradford, L. W., et al. 2005, AJ, 130, 2262

Saffe, C., Gómez, M., \& Chavero, C. 2005, A\&A, 443, 609

Skrutskie, M. F., Cutri, R. M., Stiening, R., et al. 2006, AJ, 131, 1163

Tamuz, O., Ségransan, D., Udry, S., et al. 2008, A\&A, 480, L33

Tsantaki, M., Sousa, S. G., Santos, N. C., et al. 2014, A\&A, 570, A80

ten Brummelaar, T. A., Mason, B. D., Bagnuolo, W. G., Jr., Hartkopf, W. I., McAlister, H. A., \& Turner, N. H. 1996, AJ, 112, 1180 
ten Brummelaar, T. A., Mason, B. D., McAlister, H. A., et al. 2000, AJ, 119,2403

Terziev, E., Law, N., Arcavi, I., et al. 2013, ApJS, 206, 18

Tokovinin, A. 2012, AJ, 144, 56

Turner, N. H., ten Brummelaar, T. A., Roberts, L. C., Jr., et al. 2008, AJ, 136,554
Valenti, J. A., \& Fischer, D. A. 2005, ApJS, 159, 141

van Leeuwen, F. 2007, A\&A, 474, 653

Vigil, M. L., Witte, D. J., Levan, P. D., et al. 1996, Proc. SPIE, 2819, 151

Wizinowich, P., Acton, D. S., Shelton, C., et al. 2000, PASP, 769, 315

Wu, Y., \& Lithwick, Y. 2011, ApJ, 735, 109

Wu, Y., \& Murray, N. 2003, ApJ, 589, 605 